(C) 1979 IEEE. Personal use of this material is permitted. However, permission to reprint/republish this material for advertising or promotional purposes or for creating new collective works for resale or redistribution to servers

or lists, or to reuse any copyrighted component of this work in other works must be obtained from the IEEE.

IEEE Transactions on Nuclear Science, Vol. NS-26, No. 3, June 1979

\title{
SLOW EXTRACTION FROM KEK-PS MAIN RING
}

Kuninori Endo, Yoshitaka Kimura, Shinji Mitsunobu, Shigeshi Ninomiya, Yutaka Sakamoto, Hikaru Sato, Yasunao Takeuch1 and Shuich1 Tokumoto*

\section{Summary}

Two different schemes are used for the slow beam extraction from the main ring of KEK-PS. One uses the internal target scattering under the non-resonant condition and the other the ha1f-integer resonance at $v$ $=7.5$. The extraction efficiency is higher for the $x$ resonant extraction ( $99 \%$ ) than for the non-resonant scheme $(230 \%)$. The spill feedback system are provided to suppress the intensity modulation of the extracted beam and to obtaln the sp111 duration up to 400 msec. Slow extraction also shares the beam with the internal target in both schemes in order to perform experiments on both floors at the same time.

\section{Introduction}

Typically half- and third-integer resonance extractions are being utilifzed in the alternatinggradient synchrotrons: ${ }^{1-4}$ Choice between the two depends on the feasibility of the beam handling in an accelerator. At KEK-PS a possibility of the halfinteger resonance extraction was clarified through the theoretical studies.5 6 ) The slow extraction system was based on the detailed numerical study ${ }^{6}$ and brought into operation in Nov., 1977. Since then the experimental study on the slow extraction is being continued alongside of the physics experiments.

In the meantime, the possibility using the scattered beam from the internal target?" which has been in operation since 1977, for the slow extraction was investigated ${ }^{8}$ and tried successfully last spring. As indicated by the Monte Carlo simulation the extraction efficiency is not high, but the stable slow spill with the $30 \%$ extraction efficiency is obtained. At present the following operations are ready for experiments,

- Resonant extraction

- Resonant sharing (between slow extraction and internal target)

- Non-resonant sharing using target scattering.

In all cases, the fast extraction to the bubble chamber can be parasitized at both beginning and ending of the slow extraction.

\section{Resonant Slow Extraction}

Just after reaching flat-top the horizontal tune is shifted to $\nu_{\mathrm{x}}=7.38$ within $100 \mathrm{msec}$ by increasing the current of the focusing lattice quadrupole magnets by $0.3 .3 \%$, while the vertical tune remalns unchanged. Then two iron-core extraction quadrupole magnets (EQ1 and EQ2) and the octupole magnet (0) shown In Fig.1 are excited. To get long spill up to $2400 \mathrm{msec}, \mathrm{EQ1}$ gives the ramped quadrupole field to drive the circulating particles into the half-integer resonance successively. Almost at the same time, four bump magnets (BM) shift the closed orbit outwards by $15 \mathrm{~mm}$ and five septum magnets (SM) are powered during extraction. An electrostatic septum (ESS) is held at a constant voltage meanwhile.

The resonant extraction was tried at 8 and $12 \mathrm{GeV}$. At $8 \mathrm{GeV}$, the current ripples of the lattice magnets are $23 \times 10^{-5}$ for the quadrupole magnets and $25 \times 10^{-5}$ for the dipole magnets under the operation of the dynamic filters. At $12 \mathrm{GeV}$, however, ripples are $22 \times 10^{-4}$ for both quadrupole and dipole magnets. The ripple is compensated at both energies in such a way that the spill signal from the secondary emission monitor (SEM) placed at the extraction channel is fed to excite the air-core quadrupole magnets for the ripple correction (RQ1 and RQ2). The low frequency structures lower than $100 \mathrm{~Hz}$ in the spilt beam are corrected by the feedback to EQ1.

EQ2 is not required for the $8 \mathrm{GeV}$ operation, but for $12 \mathrm{GeV}$ it is used to adjust the separatrix finely at the entrance of ESS. With EQ2 the extraction eff1ciency amounts to $99 \%$ but $v 70 \%$ without 1 t.

\section{Resonant Sharing}

Resonant conditions are held almost as same as in the previous paragraph except that the extraction begins at the lower excitation of $\mathrm{EQ1}$, since the momentum loss when scattered by the Internal target increases the horizontal tune due to the negative machine chromaticity $\left(\Delta \nu_{x} \simeq-10.3 \Delta \mathrm{p} / \mathrm{p}\right.$ at $8 \mathrm{GeV}$ and $-17.6 \Delta \mathrm{p} / \mathrm{p}$ at $12 \mathrm{GeV})$. The ${ }^{\mathrm{x}}$ sharing ratio can be controlled by changing the local bump at ESS. The minimum sharing
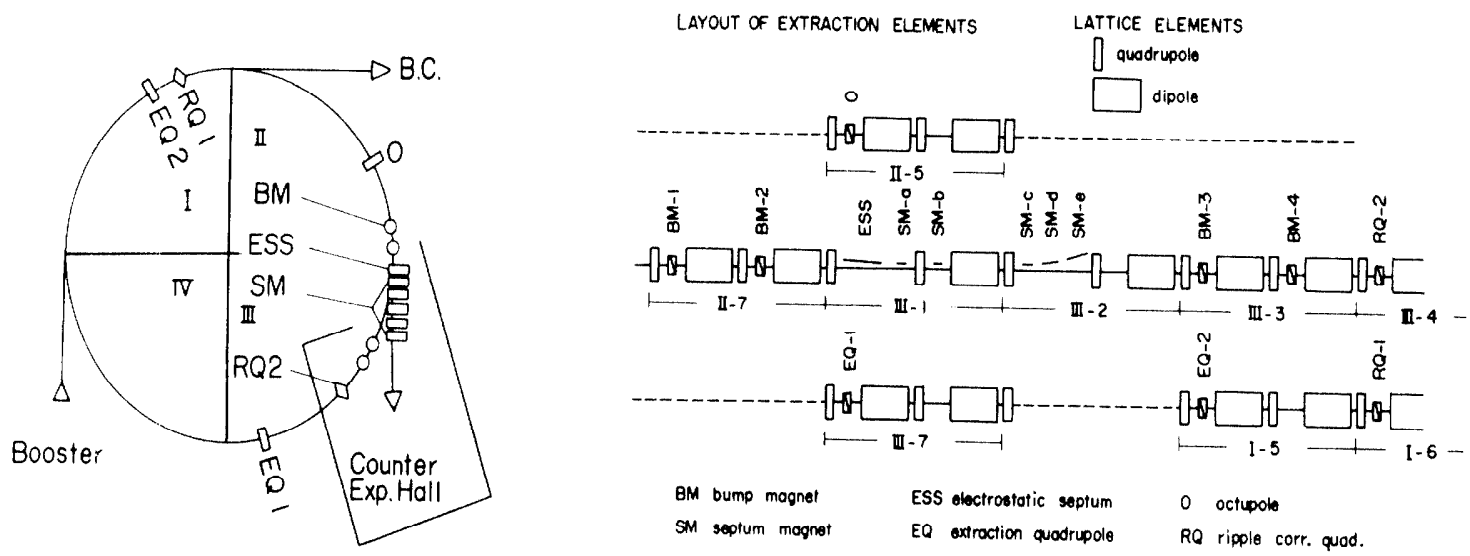

F1g.1 Layout of the extraction elements.

* National Laboratory for High Energy Physics,

Oho-machi, Tsukuba-gun, Ibaraki-ken, 300-32, Japan 
obtained to the slow extraction was $80 \%$ and the rest of the circulating beam partly interacts in the internal target and partly be lost hitting the vacuum chamber. The internal target placed at III5F is inserted so as to touch the envelope of the beam when the local closed prbit bump (target bump) is applied at this position?

\section{Non-resonant Sharing}

By shifting the beam to the internal target with the target bump mentioned above, the scattered proton increase their betatron amplitudes and some of them jump across ESS and then are extracted. This process may be ready under resonant or non-resonant excitation pattern of the lattice quadrupole magnets. Higher extraction efficiency is obtained for the non-resonant excltation. For the target material, the thin and light target such as Be is best. ' The maximum theoretical extraction efficiency is $255 \%$ but it is difflcult to obtain a flat spill at higher efficiency than $230 \%$ for the $1 \mathrm{~mm}$ Be target (Fig.2). To get uniform sp111 for both slow extraction and internal target, both amplitudes of the closed orbit bumps are controlled independently using signals from SEM and a scintillation counter (secondary particle monitor) at the internal target. Though the extraction efficiency is not high, this operation mode is useful for some experiments because the intensity modulation of the spilt beam is smaller than that of the resonant extracted beam.

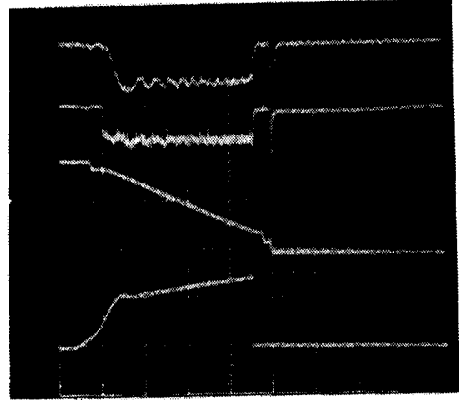

Fig.2 Non-resonant sharing between slow extraction and internal target $(1 \mathrm{~mm} \mathrm{Be})$ at $8 \mathrm{GeV}$. Top trace: Slow extracted spill, 2nd: Internal target spill, 3rd: Circulating beam intensity, bottom: Local closed orbit amplitude at ESS ( $100 \mathrm{~ms} /$ div.). Fast beams are a1so extracted at both ends.

\section{Extraction Elements}

Extraction clements are grouped into two groups such as the resonance and extraction systems. The former drives the circulating particles into resonance by controlling the betatron oscillation using the spilt beam signal. The latter system leads particles out of the main ring to the beam channel transporting them to the external target in the experimental hall.

\section{Resonance System}

Extraction quadrupoles (EQ1 and EQ2) Mainly EQ1 is excited to shift the horizontal tune close to the stop band of 7.5. Its location was determined to be III7F according to the numerical beam tracing so as to adopt the outgoing separatrix to the extraction system at IIIIF and III2F. It has enough power to move the tune from 7.38 to 7.5 . The maximum magnetic power is 6.7 $\mathrm{T} / \mathrm{m}$ with the $0.3 \mathrm{~m}$ core length. EQ2 has the same parameter as EQ1 but its role is different from EQ1. EQ2 is located at I5F.

Octupole (0) Perturbation of the octupole field shifts the horizontal tune of the beam with larger amplitude further close to $v=7.5$. Octupole field is fixed stationary during extraction and its effect on the beam is so weak that the circulating particles become slowly unstable. Its maximum magnetic power is $10^{3} \mathrm{~T} / \mathrm{m}^{3}$ with the $0.25 \mathrm{~m}$ core length. Octupole is located at II5F. This position is separated by an integral multiple of the betatron wave length from EQ1 when $1 t$ is excited.

Ripple correction quadrupoles (RQ1 and RQ2) Two aircore quadrupole magnets are designed to correct the spill structures higher than $50 \mathrm{~Hz}$ (up to $600 \mathrm{~Hz}$ ) which arises mainly from the ripple of the 12-phase rectifier of the lattice magnets. The power supplies are ac power amplifiers with the maximum rating of \pm 60 $\mathrm{V}$ and $\pm 60 \mathrm{~A}$. Inductance of the magnet including the cable is $20.3 \mathrm{mH}$.

\section{Extraction system}

Electrostatic Septum (ESS) The electrostatic septum consists of three 1 m modules and the septum is made of the $75 \% \mathrm{~W}-25 \%$ Re wires of $50 \mu \mathrm{m}$ in diameter with a spacing of $1.25 \mathrm{~mm}$. The nominal rating is $60 \mathrm{kV} / \mathrm{cm}$ for a gap of $2 \mathrm{~cm}$. The horizontal deflection is 0.47 mrad per meter at $60 \mathrm{kV} / \mathrm{cm}$ for $12 \mathrm{GeV}$.

Septum magnets (SM) Five septum magnets are used to guide the particles spilt along the separatrix to the counter experimental hall as shown in Fig.1. Their parameters are given in Table $\mathrm{I}$.

Table I Parameters of the septum magnets $\begin{array}{ccc}\text { septum } & \text { max.field lenglh } \\ \text { thickness }(\mathrm{mm}) & (\mathrm{kG}) & (\mathrm{m})\end{array}$

$\begin{array}{lrrr}\text { SM-a } & 1 & 1 & 0.9 \\ \text { SM-b } & 3 & 2 & 0.7 \\ \text { SM-c } & 15 & 8 & 1.5 \\ \text { SM-d } & 35 & 16 & 1.3 \\ \text { SM-e } & 35 & 16 & 1.3\end{array}$

Bump magnets (BM) To make the local closed orbit bump at ESS, four bump magnets are used. Amplitude and angle of the beam at the entrance of ESS can be controlled to adjust the turn separation during extraction. The maximum deflection angle of single BM is 4 mrad at $12 \mathrm{GeV}$ and the maximum amplitude of the bump is $20 \mathrm{~mm}$ with angle of $-1 \mathrm{mrad}$ at ESS.

\section{Spill Feedback Control for Resonant Extraction}

In order to get unfform spill during extraction, the spill signal obtained from SEM is compared with the reference signal, which is generated from the circulating beam intensity to get the desired spill duration, and the difference signal is inverted and then added to the input signal of the power supply for EQ1. The frequency response of EQ1. decreases for higher frequency than $100 \mathrm{~Hz}$, and RQ plays a complemen-

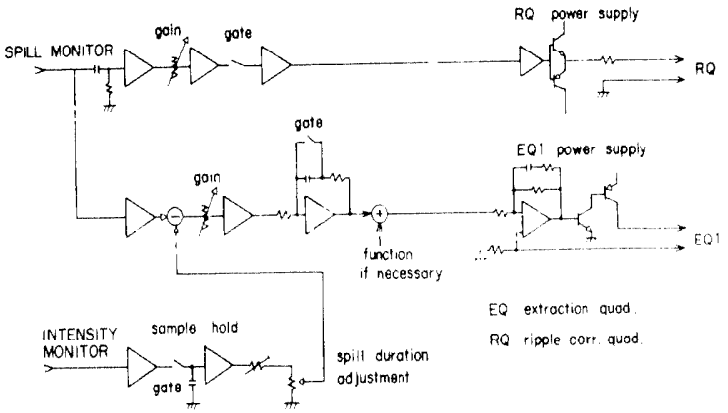

Fig. 3 Schematic diagram of the spill feedback system. 
tal role in the spill control. A schematic diagram of the spill feedback system 1s shown in Fig.3. After correcting the spill structure with EQ1 and $R Q$, the high frequency structure of 2 $23 \mathrm{kHz}$ is observed as shown in Fig.4. Up to $U 1 \mathrm{kHz}$ it is possible to correct the sp111 structure by the present spill feedback system. The residual structure has some relationship with the debunching process of the RF bunches in addition to an instability of the spil1 feedback system. A much higher frequency structure corresponding to the revolution frequency and its harmonic is also observed with the gas scintillation counter (Fig.5). Correction of high frequency structure is the urgent problem.

\section{Beam Performance}

Extractec beam intensity was measured with SEM which was calibrated at the fast extraction channel comparIng with the same intensity monitor as used in the main ring for the measurement of the circulating beam. The $99 \%$ efficiency was obtained when a part of chromaticity was compensated. Unless chromaticity was compensated, a part of the circulating beam was lost in the main ring when the horizontal tune was shifted close to the halfinteger resonance and the extraction efficiency remained at $66 \%$. However, the sextupole magnets were not excited to the level of the full compensation of chromaticity. It was found that the strong sextupole field led to the growth of the extracted beam emittance and the vertical deflection of the extracted beam.

The emittance of the extracted beam was measured with SWIC (segmented wire ton chamber) by changing the parameters of the quadrupole magnets of the extracted beam channel. Measurement gave $18 \mathrm{~mm}$ mrad horizontal$1 \mathrm{y}$ and $v_{0} \mathrm{Tmm}$ mrad vertically. These values are larger than the expected ones theoretically by a factor of two. Horizontally it reflects the fact that the turn separation is not controlled precisely against the tune fluctuation and the momentum distribution in the beam. Vertically, however, the coupling between the horizontal and vertical motions in the main ring will occur in the sextupole fleld (arising from dipole magnets and correction sextupole magnets) due to the distortion of the closed orbit and result in the vertical blowup.

\section{Acknowledgement}

The authors wish to acknowledge the continual cooperations of the members of the beam channel group for the measurements of the beam performances. They also express thanks to $\mathrm{Mr} . \mathrm{K}$. Sato for his contribution to the measurement of the beam profile at the electrostatic septum and to the members of the main ring power supply group for preparing the current patterns. Finally they want to thank Prof. T. Kame1 for his steady interest during the course of the work.

\section{References}

\footnotetext{
M. Bell and W. Kubischta, "Simulation of Simulta-
}
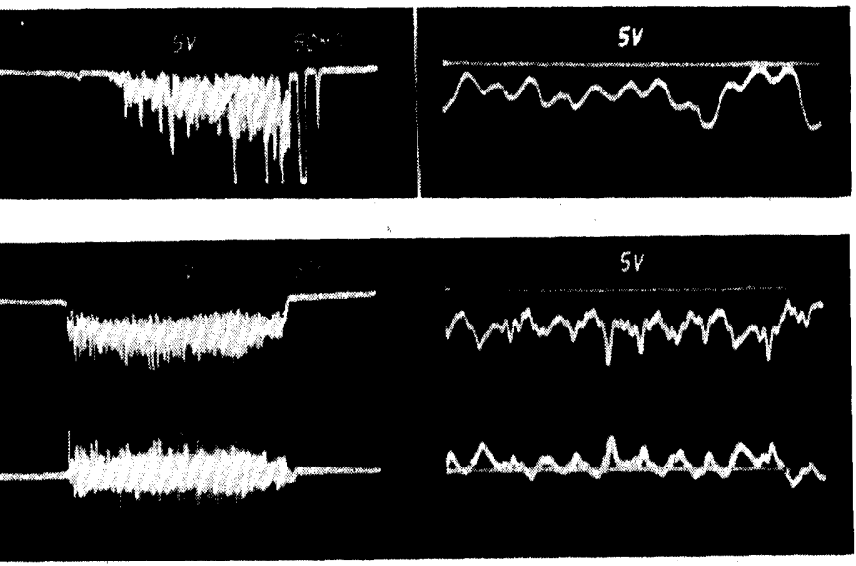

$50 \mathrm{~ms} / \mathrm{div}$

$2 \mathrm{~ms} / \mathrm{div}$

ill structure observed with SFM. a) Spill structure when corction is not applied. b) Spill structure corrected with both 1 and $\mathrm{RQ}$. Top trace: slow beam spill (SEM signal), bottom ace: current of $\mathrm{KQ}$.

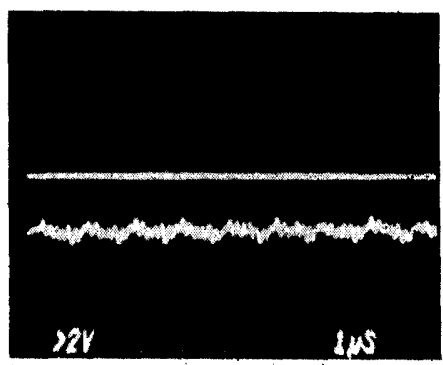

Fig.5 Beam spill observed with the gas scintillation counter ( $1 \mu \mathrm{s} / \mathrm{div}$ ).

neous Beam Sharing between Internal Targets and Slow Ejection at the CPS", Proc. 9th Int. Conf. on High Energy Acc. (SLAC, 1974) p.467.

2) L.N. Blumberg, M.Q. Barton, G.W. Bennett, J.D. Fox, J.W. Glenn, H.C.H. Hsich, R.J. Nawroaky, and A.V. Soukas, "Initial Performance of the AGS S1ow External Beam", IEEE Trans., NS-16 (3) (1969) 234.

3) H.T. Edwards, W.W. Lee, J.D. McCarthey, S. Ohnuma and L.C. Teng, "Half-Integer Resonance Extraction for the NAL Accelerator", IEEE Trans. NS-20 (3) (1973) 424.

4) Y. Baconnier, P. Faugeras, K.H. Kissler, B.de Raad and W. Scandale, "Extraction from the CERN SPS", IEEE Trans., NS-24 (3) (1977) 1434.

5) T. Suzuki and S. Kamada, "Theory of Half-Integral Resonant Extraction", KEK-76-7 (1976).

6) K. Endo and C. Steinback. "Half Integer Resonant Extraction from Main Ring of KEK Proton Synchrotron", KEK-77-13 (1977).

7) K. Endo, S. Ninomiya and Y. Takeuchi, "Servo-Spill System for KEK Internal Target", Proc. 2nd Symp. on Accelerator Science and Technology (INS, 1978) p. 179 .

8) C. Stelnback and K. Endo, "Simultaneous Sharing of Slow Extraction and Internal Target", KEK-77-18 (1978). 